\title{
Supernova Remnants in the Large Magellanic Cloud:
} A Multiwavelength Study of Energetics and Environments

\author{
Rosa Murphy Williams, You-Hua Chu, and John R. Dickel \\ Astronomy Department, University of Illinois, Urbana, IL 61801, USA
}

R. Chris Smith

Cerro Tololo Inter-American Observatory, La Serena, Chile

\begin{abstract}
.
We analyze optical, radio and X-ray data from supernova remnants (SNRs) in the Large Magellanic Cloud (LMC) in order to make a systematic study of a full sample at a known distance. By this multiwavelength examination of SNRs from various progenitors and environments, we will build a comprehensive picture of the evolution of SNR structure and energetics.
\end{abstract}

\section{Introduction}

The Large Magellanic Cloud (LMC), provides an ideal setting for the study of supernova remnants (SNRs) at a common distance and with little interstellar absorption. We analyze optical, radio and X-ray data from these remnants, in order to make a systematic study of the full LMC sample. These data are being used to investigate such questions as the partitioning among thermal, kinetic, possibly magnetic, and relativistic-particle energies; the pressures from different components within a remnant; the density and clumpiness of the gas inside and immediately outside the remnants; and the effects of local interstellar gas on SNR development.

\section{Available Datasets}

$\mathrm{X}$-ray images and spectra are available through archival and proprietary data from the ROSAT and $A S C A$ satellite instruments. X-ray images show the distribution of million-degree gas within an SNR, providing information on shock structure for comparison with details of the local environment, as well as the possible presence of internal cloudlets. X-ray spectra address questions of internal temperatures, densities, hot gas (thermal) pressures, and energetics.

Long-slit echelle spectra for most LMC SNRs have been obtained through observations with the $4 \mathrm{~m}$ telescope at Cerro Tololo Inter-American Observatory (CTIO). Echelle spectra provide velocity information via the Doppler shifts of the $\mathrm{H} \alpha$ line emission. These spectra give expansion and shock velocities for an SNR, which can be used in the derivation of ages and kinetic energies. 
A variety of optical images have been made available by the Magellanic Cloud Emission-Line Survey, using the Curtis Schmidt telescope at CTIO. Additional images taken with the CTIO $1.5 \mathrm{~m}$ and $4 \mathrm{~m}$ telescopes are also available. Optical images show the distribution of cooler gas in the shell of the SNR, with different ions being produced by different shock conditions. $\mathrm{H} \alpha$, [S II], and [O III] images can be combined for a detailed picture. The surface brightness of the $\mathrm{H} \alpha$ emisson, combined with estimates of the shell geometry, can give approximate figures for particle density; from this, the shell mass, kinetic energy, and cool gas (thermal) pressure can be derived.

Approximately half of the known LMC SNRs have been observed at radio wavelengths using the Australia Telescope Compact Array (ATCA). Radio images show the location of synchrotron emission, which is generated by the motion of relativistic electrons around magnetic field lines compressed behind the shock front. From the spectral index of the radio observations, we can derive the energy and pressure of magnetic fields within a remnant.

\section{The Big Picture}

By analyzing the data for each SNR at different wavelengths, we can build a comprehensive picture of its structure and energetics. Combining the data with observations of the SNR's immediate surroundings provides clues to the effects of local conditions on remnant development, as with a break-out into a local cavity, or the sweeping up of cloudlets into the SNR interior. Because the LMC contains a large sample of SNRs with different initial conditions, in a wide variety of environments, we compare the remnants to one another in order to determine the relative influence of various factors on SNR evolution, and, in return, the effects of the SNRs on their surroundings. Examples of such analyses have been published for the colliding remnants DEM L 316 (Fig. 1a; Williams et al. 1997) and two SNRs, N $11 \mathrm{~L}$ and N 86, which show breakout structures (Fig. $1 \mathrm{~b}$; Williams et al. 1999). We hope by these means to gain a clearer picture of the role SNRs play in the structure and evolution of the interstellar medium.
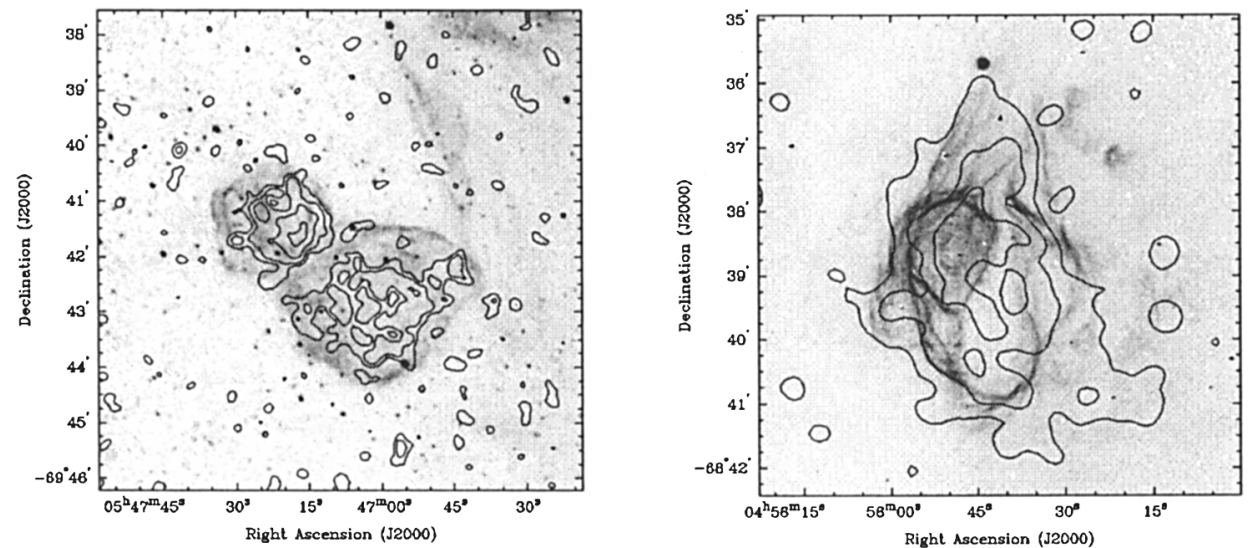

Figure 1. $\mathrm{H} \alpha$ images of (a) DEML 316 (b) N 86 with X-ray contours. 


\section{References}

Williams, R. M., et al. 1997, ApJ, 480, 618

Williams, R. M., et al. 1999, ApJ, 514, 798

\section{Discussion}

Nolan Walborn: Is there velocity information which supports the interpretation at the "double SNR" as two separate expansions, as opposed to a single bipolar one? The latter seems more probable from the morphology to me. The two lobes have similar, mirror image shapes, and the larger one has a lower surface brightness in X-rays. It must be very improbable to get such a configuration from two unrelated events. I suggest that a model with a very dense equatorial disk should be considered.

Williams: In fact, echelle velocity spectra show DEM L 316 as two separately expanding shells. In addition, were DEM L 316 the result of a bipolar outflow, the two shells must either be precisely perpendicular to the line of sight - an unlikely coincidence - or else show different radial velocities at the extreme ends. Instead, both converge to the object's $245 \mathrm{kms}^{-1}$ heliocentric velocity. Futher evidence for interaction is provided by the coincidence of the $\mathrm{X}$-ray brightening of one shell with the outer [O III] edge of the other, as well as an observed change in the radio polarization between the shells. Finally, a bipolar expansion would require the dense equatorial disk which you mentioned; we have looked for evidence of such material in H I maps provided by Stavely-Smith and Kim, but find no local increase at the juncture between the shells. A full treatment of these arguments is in Williams et al., ApJ, 480, 618 (1997). 